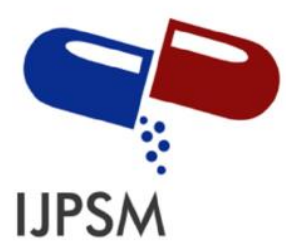

Karishma Chordiya et al, Int. Journal of Pharmaceutical Sciences and Medicine (IJPSM),

Vol.6 Issue. 4, April- 2021, pg. 83-91

ISSN: 2519-9889

Impact Factor: 3.426

\title{
Role of Nanoparticles in the Management of Diabetes
}

\author{
Karishma Chordiya; Hritik Patwa; Sanjay G. Walode; Vibhavari M Chatur* \\ Rasiklal M. Dhariwal Institute of Pharmaceutical Education and Research, Chinchwad, Pune
} DOI: 10.47760/ijpsm.2021.v06i04.007

\begin{abstract}
One of the significant purposes of nanotechnology in the field of medical science is the utilization of nanotechnology in drug delivery to target the tissue for the treatment of disease like diabetes. The biologically compatible nanoparticles with biological, chemical, physical properties may be useful to enhance and overcome the limitations which are caused in the use of traditional medicine system by boosting the rate of drug release ,enhancing drug solubility, and improving the diffusion and distribution of drugs. In this review, we have highlighted on the term diabetes and its different types. Diabetes is becoming an epidemic, more than 366 millions of people around the world are affected. This, article also focuses on the role of nanoparticles in the management of diabetes also its different types of as well as its applications and uses in the treatment of diabetes.
\end{abstract}

Keywords: Diabetes, Nanoparticles, Insulin, Nanocarrier, Nanomedicine.

\section{Nanoparticles:}

\section{Introduction:}

Nanotechnology signifies to an developing area of science which contains growth and synthesis of several nanomaterials . They are the The solid particles or particulate dispersions having a size range $1-100 \mathrm{~nm} .^{[1]} \mathrm{In}$ nanoparticle matrix, the drug is attached ,encapsulated, entrapped or dissolved. Depending on the preparation method, they may be obtained in the form of nanocapsules, nanospheres or nanoparticles. The drug which is evenly and physically dispersed in a matrix system is called as nanospheres the system wherein the drug is enclosed to a cavity bounded by a unique polymer membrane is called as Nanocapsules. In recent years, due to the capability to circulate for elongated period time target a specific organ, their capacity to supply peptides, genes and proteins and in gene therapy as a carrier, long-circulating particles had been used as potential drug delivery system. Long-circulating particles are known as biodegradable polymeric nanoparticles, specifically that coated by hydrophilic polymer i.e polyethylene glycol (PEG). ${ }^{[2]}$

To control surface properties, particle size and delivery of pharmacologically active agents so as to get the sitespecific action of drug at dose regimen and therapeutically optimal rate are main aim in developing nanoparticles as a delivery system. With unique advantages of liposomes such as targeting to site of action , preserving drugs from degrading and reducing side effects and toxicity, liposomes had been used as a potential carriers ,the applications of liposomes are limited as a result of inherent problems such as poor storage stability ,low encapsulation efficiency and fast leakage of hydrophilic drug in presence of blood components. In contrast, polymeric nanoparticles gives some special advantages over liposomes. For example, they reserves useful controlled release properties and helps in increasing the stability of proteins or drugs. ${ }^{[2]}$ 


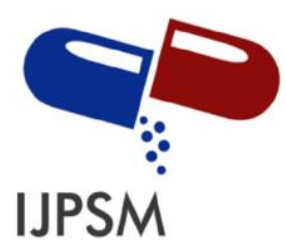

Karishma Chordiya et al, Int. Journal of Pharmaceutical Sciences and Medicine (IJPSM),

Vol.6 Issue. 4, April- 2021, pg. 83-91

ISSN: 2519-9889

Impact Factor: 3.426

\section{Definition: ${ }^{[3]}$}

Nanoparticles are defined as a small tiny particles having a size range of 1-100nm. Nanoparticles are broad class of materials which comprises of specific substances, that have dimension less than $100 \mathrm{~nm}$.

Depending on shape the nanoparticles may be zero dimensional, one dimensional, two dimensional, three dimensional.

Nanoparticles are complex molecules itself and thus consist of three layers i.e 1) the surface layer 2) the shell layer 3) the core.

\section{Classification of Nanoparticles: ${ }^{[4]}$}

Nanoparticles are mainly classified in to three classes.

\section{One dimension nanoparticles}

For decades One dimensional system had been used. Monolayer / Thin films (1-100 nm) is now common place in the arena of solar cells recommending, different technological applications, for instance biological and chemical sensors, magneto-optic and optical device, information storage systems, fiber-optic systems.

\section{Two dimension nanoparticles}

Carbon nanotubes

\section{Three dimension nanoparticles}

Dendrimers, Fullerenes (Carbon 60), Quantum Dots

\section{Another classification based on physical and chemical characteristics:}

Carbon-based NPs, Metal NPs, Ceramics NPs,, Semiconductor NPs, Polymeric NPs, Lipid-based NPs, Alloy NPs, Magnetic NPs.

\section{Applications of Nanoparticles: ${ }^{[5]}$}

1. Magnetic resonance imaging (MRI)

2. Optical imaging

3. in drug and gene delivery

4. Neurodegenerative diseases

5. Cancer

6. HIV/AIDS

7. Ocular diseases

8. Respiratory diseases

\section{Diabetes:}

\section{Introduction: ${ }^{[11]}$}

Diabetes is a chronic disease that causes increase of sugarlevel in blood. Organs like nerves, eyes, kidneys and other organs can be damaged due to untreated high blood sugar from diabetes.

Diabetes Mellitus (DM) has been recognized to human for over 2000 Years. It is probable to develop one of the greatest predominant and economically significant diseases of the $21^{\text {st }}$ century. Diabetes mellitus is a cluster of 


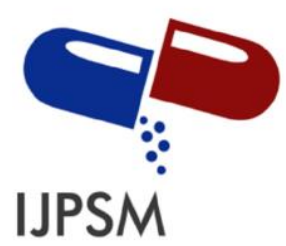

Karishma Chordiya et al, Int. Journal of Pharmaceutical Sciences and Medicine (IJPSM),

Vol.6 Issue. 4, April- 2021, pg. 83-91

ISSN: 2519-9889

Impact Factor: 3.426

metabolic disorder described by a entire deficiency of insulin, a relative absence of insulin, or insulin resistance, that later lead to hyperglycemia. Diabetes mellitus has touched outbreak ratios in many countries. the occurrence of type 2 diabetes mellitus is expected to rise intensely across the coming years. worldwide, it is likely that the number of persons having diabetes mellitus will increase to $35 \%$ by 2025 . the rise in occurrence of diabetes mellitus is because of three impacts: lifestyle, age and background.

\section{Definition: ${ }^{[6]}$}

When the pancreas is no longer able to make insulin or the condition in which body cannot make good use of insulin, such condition is called as diabetes.

\section{Types of Diabetes:}

There are majorly two types of diabetes

a) Type 1 diabetes

b) Type 2 diabetes

\section{Type 1 diabetes: ${ }^{[7]}$}

Type 1 diabetes is also termed or known as insulin dependent diabetes. Mostly it develops in children or young adult, it can affect people at any age. In the type 1 diabetes, body's defence system attacks the cells of pancreas which produces insulin, as a result the production of insulin is very less or there is no production. In some cases, the organ is damaged and doesn't make insulin. This type of diabetes can be caused by your genes. If there is problem with cells in your pancreas that makes insulin, type 1 diabetes takes place. There are chances of death, if the patient is suffering from type 1 diabetes and do not have access to insulin.

\section{Symptoms Of Type 1 Diabetes :}

1) Recurring thirst and dry mouth

2) Increase in frequency of urine

3) Sudden weight loss

4) Slow healing wounds

\section{Type 2 diabetes: ${ }^{[8]}$}

Type 2 diabetes has been increasing steadily all over the world, it is a chronic metabolic disorder. Currently it is rapid developing an widespread in several countries of the world. It is expected that the number of people affected will become double in the next decade. It is generally characterized by insensitivity of insulin resistance, less insulin production and eventual pancreatic beta cell failure. Hence, this leads to decline in transport of glucose into the muscle cells, fats cells and liver. Breakdown of fat is increased with hyperglycemia, this leads to impaired alpha cell function which lead to type 2 diabetes.

\section{Symptoms Of Type 2 Diabetes :}

1. Increased thirst

2. Frequent urination

3. Increased hunger

4. Unintended weight loss

5. Fatigue

6. Blurred vision 


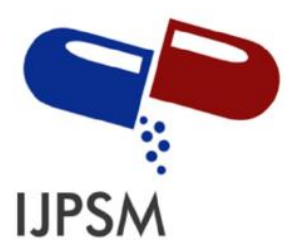

Karishma Chordiya et al, Int. Journal of Pharmaceutical Sciences and Medicine (IJPSM),

Vol.6 Issue. 4, April- 2021, pg. 83-91

ISSN: 2519-9889

Impact Factor: 3.426

\section{Current types of treatment for Diabetes: ${ }^{[9]}$}

\section{Diabetes management:}

The management of diabetes is very necessary. The diabetes impact your full body. The following steps are needed to maintain your risk factors under limit and in normal range

- By taking given prescribed medicines, enhancing your activity level and taking a diet plan, maintain your blood glucose levels nearby to normal.

- As possible as maintain your HDL and LDL levels i.e blood cholesterol and triglyceride levels to normal values.

- Keep your blood pressure under control. It should be not more than $140 / 90 \mathrm{mmHg}$

\section{Diabetes treatment :}

Treatments for diabetes based on type of diabetes.

- Type 1 diabetes: patient should take insulin every day, if having this type of diabetes. pancreas no longer produces insulin.

- Type 2 diabetes: treatments can comprise medications, insulin and lifestyle changes like as weight losing, producing healthy food selections and being extra physically active.

\section{Oral medicines and insulin function these ways to treat diabetes:}

- Promotes your pancreas to produce and release extra insulin.

- Reduces the delivery of glucose from your liver.

- Stops the breakdown of carbohydrates in stomach or intestines so as tissues are extra susceptible to insulin.

- Facilitates to free your body of glucose via enhanced urination.

\section{Oral Medications To Treat Diabetes $:^{[9]}$}

Across 40 medicines have been approved by the FDA for the healing of diabetes.

The medication used in the treamtment of Diabetes.

Sulfonylureas: These drugs reduces blood glucose by triggering the pancreas to release extra insulin. Examples contain glimepiride (Amaryl $\left.{ }^{\circledR}\right)$, glyburide (Micronase ${ }^{\circledR}$, DiaBeta $\left.{ }^{\circledR}\right)$ and glipizide (Glucotrol ${ }^{\circledR}$ )

Glinides (also called meglitinides): These drugs decrease blood glucose by causing the pancreas to release more insulin. Examples include nateglinide (Starlix ${ }^{\circledR}$ ) and repaglinide (Prandin $\left.{ }^{\circledR}\right)$.

Biguanides: These drugs lowers how much glucose the liver makes. It similarly enhances in what way insulin works in the body, and brakes down the conversion of carbohydrates into sugar. Example - Metformin (Glucophage ${ }^{\circledR}$ ) .

Alpha-glucosidase inhibitors: These drugs decrease blood glucose by postponing the breakdown of carbohydrates and decreasing glucose absorption in small intestine. Example acarbose (Precose®). 


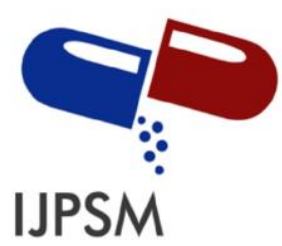

Karishma Chordiya et al, Int. Journal of Pharmaceutical Sciences and Medicine (IJPSM), Vol.6 Issue. 4, April- 2021, pg. 83-91

ISSN: 2519-9889

Impact Factor: 3.426

Thiazolidinediones: These drugs enhance the method insulin works in the body by permitting extra glucose to go into muscles, fat \& liver. Examples contain rosiglitazone (Avandia $\left.{ }^{\circledR}\right)$ \& pioglitazone (Actos ${ }^{\circledR}$ ).

GLP-1 analogs (also known as incretin mimetics or glucagon-like peptide-1 receptor agonists): These drugs boost the release of insulin, decrease glucose discharge from the liver after meals and postpone food emptying from the stomach. Examples include exenatide (Byetta $\left.{ }^{\circledR}\right)$, dulaglutide (Trulicity®), albiglutide (Tanzeum ${ }^{\circledR}$ ), semaglutide (Rybelsus $\left.{ }^{\circledR}\right)$.

DPP-4 inhibitors (also known as dipeptidyl peptidase-4 inhibitors): These drugs support pancreas liberate more insulin after meals. This moreover lowers the quantity of glucose released by the liver. Examples include alogliptin (Nesina $\left.{ }^{\circledR}\right)$, sitagliptin (Januvia $\left.{ }^{\circledR}\right)$, and linagliptin (Tradjenta $\left.{ }^{\circledR}\right)$.

SGLT2 inhibitors (also known as sodium-glucose cotransporter 2 inhibitors): These drugs act on your kidneys to eliminate glucose in your body via your urine. Examples contain canagliflozin (Invokana®), empagliflozin (Jardiance®), dapagliflozin (Farxiga $\left.{ }^{\circledR}\right)$.

Bile acid sequestrants: These drugs reduce cholesterol and blood sugar amounts. Examples comprise colestipol (Colestid $\left.{ }^{\circledR}\right)$, colesevelam (Welchol ${ }^{\circledR}$ ) and cholestyramine (Questran $\left.{ }^{\circledR}\right)$.

Dopamine agonist: This medication drops the quantity of glucose released by the liver. Example bromocriptine (Cyclocet $\left.{ }^{\circledR}\right)$.

Various oral diabetes medicines might be used in combination or with insulin to accomplish the excellent blood glucose control. Various of the above medicines are available as a combination of two medicines in a one pill. The GLP-1 agonist semaglutide (Ozempic ${ }^{\circledR}$ ) and lixisenatide (Adlyxin $\left.{ }^{\circledR}\right)$ are other available as injectable medicines.

\section{Insulin medications to treat diabetes:}

There are various forms of insulins for diabetes.

Rapid-acting insulins: insulin glulisine (Apidra $\left.{ }^{\circledR}\right)$, insulin lispro (Humalog $\left.{ }^{\circledR}\right)$ and insulin aspart (NovoLog $\left.{ }^{\circledR}\right)$.

Short-acting insulins: insulin regular (Humulin $R \circledast)$.

Intermediate-acting insulins: neutral protamine Hagedorn(NPH) insulin.

Long-acting insulins: insulin glargine ( Basaglar ${ }^{\circledR}$, Lantus ${ }^{\circledR}$, Toujeo () ), insulin detemir (Levemir $\left.{ }^{\circledR}\right)$ and insulin degludec (Tresiba $\left.{ }^{\circledR}\right)$.

\section{Nanoparticles Used In Treatment Of Diabetes: ${ }^{[12]}$}

The huge problem to limit the diabetes is the improper administration of insulin through which the medical nanotechnology obtained enhanced to insulin delivery. Throughout the previous two decades, researchers which deal with the improvement medical factor discovered that, drug delivery is a main part of the medical development. With this respect, a broad range of drug delivery systems were recognized. These systems will improve stability and drug therapy concentration in objective tissue, additionally to those large term redistribution and release of drug assisted at the target site. The occurrence of drug administration is too decreased and enhances patient comfort. Oxidative pressure is one of the diabetes problems that triggered a interruption in wound healing which is a familiar complication in diabetic patients. It may be cured by the help 


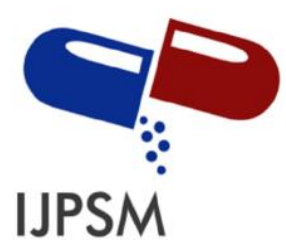

\section{Karishma Chordiya et al, Int. Journal of Pharmaceutical Sciences and Medicine (IJPSM), Vol.6 Issue. 4, April- 2021, pg. 83-91}

ISSN: 2519-9889

Impact Factor: 3.426

of some nanoparticles (aluminum oxide, and cerium oxide, gold, vanadium ,zinc) which work as an ROS absorbent.

Throughout the previous few years, numerous techniques have been suggested for non-offensive examining of blood glucose and that can be made promising by nanotechnology. Several types of nanoparticles are currently investigated for insulin supply in diabetes treatment such as,

Polymeric biodegradable nanoparticles

Ceramic nanoparticles

Polymeric micelles

Dendrimer

Liposomes

\section{Polymeric nanoparticles: ${ }^{[10]}$}

Polymeric nanoparticles are observed to be helpful and efficient across traditional oral and intravenous administration techniques. These are biodegradable polymers bounded by nanoporous membrane and are utilized as transporters of insulin. $\mathrm{pH}$ change expand the polymer system causing release of insulin. Copolymers such as $\mathrm{N}$, N-dimethylaminoethyl methacrylatem, polyanhydrides, polyurethanes, polyacrylic acids and polyacrylamide are being examined for these applications.

Cytotoxicity experiments indicated that the drug release from spherical nanosphericals strengthened and does not damage the cells . Polymeric nanoparticles possess important material for oral and intravenous forms with respect to the functioning and effectiveness, therefore polymeric nanoparticles with exclusive management may be benefited in a specific condition in distribution of high concentrations of pharmacological agents.

The past researches suggest that, these nanoparticles might be the perfect candidates for insulin therapy and insulin delivery. Polymeric nanoparticles are used as transporters of insulin.

\section{Ceramic nanoparticles: ${ }^{[10]}$}

Ceramic nanoparticles are comprised of calcium phosphate ,silica, alumina or titanium. The ceramic nanoparticles have the several advantages such as easy preparation, great biocompatibility, extra small size and high stability.

These particles can efficiently guard drug molecules compared to denaturation arises by changing the external $\mathrm{PH}$ and temperature.

Ceramic nanoparticles by environmental changes not influenced rise or changes in pore. Core of calcium phosphate nanoparticles are employed as carriers of insulin. for oral insulin delivery, calcium phosphate nanoparticles might be used.

\section{Oral delivery: ${ }^{[11]}$}

To overcome with the problem of painful subcutaneous injections, oral insulin administration is believed to be the best suitable process for the treatment of diabetes mellitus. Gastric enzymes are main obstacle for insulin when administered orally as they destroy insulin in the stomach. So, it should be enclosed in a matrix so as to get safeguarded from the harsh environment in the stomach. This may be accomplished by means of mixture of calcium phosphate-polyethylene glycol-insulin with casein. polymeric nanoparticles are filled with insulin for oral delivery of insulin were utilised in the type of pellets in animal study.

For the oral insulin administration, polymethacrylic acid, $\mathrm{N}$-isopropylacrylamide, polyethylenimine, poly (- $\varepsilon-$ caprolactone), poly (isobutylcyanoacrylate), are several polymeric nanoparticles which are being used. 


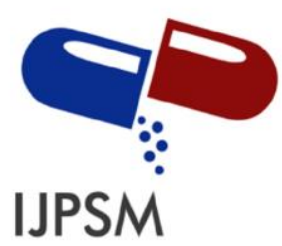

\section{Karishma Chordiya et al, Int. Journal of Pharmaceutical Sciences and Medicine (IJPSM), Vol.6 Issue. 4, April- 2021, pg. 83-91}

ISSN: 2519-9889

Impact Factor: 3.426

For the management of type 2 diabetes, chitosan nanoparticles are believed to be most effective for inhalation delivery system. Insulin is a water loving drug i.e., hydrophilic drug; so, it cannot diffuse via intestinal epithelium. Hence, for absorption of insulin, chitosan has been used as the diffusion enhancer. Encapsulation of insulin in the covering of carboxylated chitosan attached poly(methylmethacrylated) nanoparticles has enhanced the efficiency of insulin delivery.

\section{Inhalable nanoparticles: ${ }^{[12]}$}

There are various inhaler systems employed to distribute inhaled products for instance dry powder formulations and solution. Related to the gastrointestinal tract, it recommends the benefits of slight environment for instance low enzyme concentrations and neutral $\mathrm{pH}$. By means of encapsulating the insulin inside the nanoparticles, the dry powder preparation of insulin may be inspired into the lungs. This process blocks insulin degradation hence permitting direct delivery of the insulin particles to the bloodstream. It has several restrictions such as patients need regular lung function tests hence being an costly therapy.

\section{The use of antioxidant properties of nanoparticles in the treatment of diabetes: ${ }^{[10]}$}

Diabetes rises the level of free radicals and decreases the antioxidant protective mechanism might produce harm to cellular organelles, enzymes, rise in lipid peroxidation and enhance insulin resistance .Earlier studies showed that some of the nanoparticles may be used as a target drug corresponding to their role that decreases the problems of diabetes and may aid to recover diabetes and improved insulin secretion. A few of important elements are unique and comprise antioxidant properties. Their influences have been believed in bettering diabetes. The usage of components at the nanoscale and particular targets might be effective management of diseases for instance diabetes; e.g. the nanoparticles of aluminum, cerium ,vanadium, zinc, and gold. Vanadium results on type 1 and type 2 diabetes via the decreasing plasma concentration of glucose, the standardization of plasma lipid levels, improving sensitivity to insulin and insulin mimicking results.

zinc nanoparticles enhance glucose tolerance, rising of serum insulin and reduction of blood glucose . The oral usage of the gold nanoparticles as the features of antioxidant apply their performance by inhibition of creation of free radicals, clearing free radicals. These types of nanoparticles utilize the strict limit on the anti-oxidant enzymes for instance GSH, SOD, GPX and catalase, producing the inhibition of lipid peroxidation and similarly prevent the production of free radicals in hyperglycemia. Hence they might be valuable in healing diabetes.

\section{Glucose Nanosensors: ${ }^{[1]}$}

Fluorescence glucose sensors might offer a nonstop glucose reading by staying embedded into detachable wireshaped subcutaneous or intravenous tubes along with other types of implanted structures, for instance capsules, microbeads, nano-optodes, microcapsules, or capillary tubes. Nanosensor expressing plants may additionally be used to judge glucose flux variances between cells, invertase-mediated sucrose hydrolysis in vivo, transport of assimilates to roots, and glucose flux in mutants influenced in sugar transport, metabolism, and signaling.

Fluorescence glucose-sensing methods, that are under progress, recommend four probable advantages above commercially employed continuous glucose monitoring skills:

(1) Bigger sensitivity to low concentrations of glucose.

(2) No requirement to implant any transmitter or a power supply for wireless communication of glucose data.

(3) Fewer essential to recalibrate in answer to local tissue reactions across the sensor. 


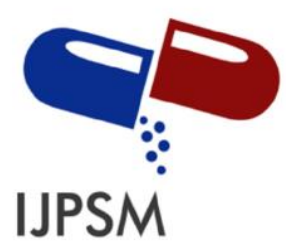

Karishma Chordiya et al, Int. Journal of Pharmaceutical Sciences and Medicine (IJPSM),

Vol.6 Issue. 4, April- 2021, pg. 83-91

ISSN: 2519-9889

Impact Factor: 3.426

(4) The opportunity of making sensors that work very precisely in the hypoglycemic range by means of binding proteins with disassociation constants in range.

\section{Use Of Nanoparticles In Treatment Of Diabetes ${ }^{:[11]}$}

Buccal insulin

Oral insulin

Poymeric NanoParticles for parenteral insulin administration

Insulin delivery through inhalable nanoparticles

Intranasal insulin delivery

Transdermal insulin

Biological micro electro mechanical systems for insulin Delivery

Glucose nanosensors

Nanoparticle-based ocular drug delivery systems

Implantable nanomedical device, which comprises pancreatic beta cells from animals

Forms of Nanoparticles available in market for Diabetes: ${ }^{[13]}$

(GLP-1)-based nanomaterials: Liposomes, Gold nanoparticle.

(GLP-1)-based nanomaterials / nano-DPP4 inhibitors: Polymeric, Chitosan, Porous silicon.

Nano-drug carriers: Polymeric, Niosomes, Nanospheres, Chitosan.

Metal NanoParticles: Zinc oxide, silver.

\section{Conclusion :}

As we have seen from above article, the diabetes is fastly growing epidemic affected in many countries of world. The influence of nanotechnology on medicine is rising day by day. Though the knowledge of nanomedicine is even in its early stages, it has main probable applications in curing diabetes. Use of nanoparticles plays a major role in treatment of diabetes. Various types of nanoparticles like polymeric biodegradable nanoparticles, ceramic nanoparticles, polymeric micelles, dendrimer, are currently under study for insulin supply in diabetes management. Nanoparticles have better stability, better oral absorption and good ability to reach target site. Their therapeutic efficacy is also better. Oral insulin in specific might prove to be hopeful, The medical functions for nanotechnology are huge and might give medicine, containing the healing of diabetes, an totally new viewpoint. 


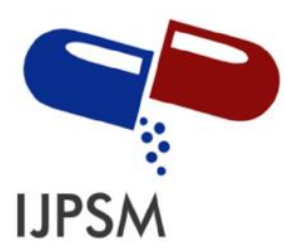

Karishma Chordiya et al, Int. Journal of Pharmaceutical Sciences and Medicine (IJPSM),

Vol.6 Issue. 4, April- 2021, pg. 83-91

ISSN: 2519-9889

Impact Factor: 3.426

\section{References}

[1]. Saba Hasan. A Review on Nanoparticles: Their Synthesis and Types. Research Journal of Recent Sciences, 2015;4:1-3.

[2]. VJ Mohanraj, Y Chen. Nanoparticles - A Review. Tropical Journal of Pharmaceutical Research, 2006;5(1):561-573.

[3]. Khan Ibrahim, Saeed Khalid, Khan Idrees. Nanoparticles: Properties, applications and toxicities. Arabian Journal of Chemistry, 2017; 12:908-931.

[4]. Bhatia Saurabh. Natural Polymer Drug Delivery Systems. Springer International Publishing Switzerland,2016:40.

[5]. Murthy Shashi K. Nanoparticles In Modern Medicine: State Of The Art And Future Challenges. International Journal of Nanomedicine, 2007; 2(2):129-141.

[6]. International Diabetic Federation: What Is Diabetes.

[7]. Gregory Justin M, Moore Daniel J, Simmons Jill H. Type 1 Diabetes Mellitus. Pediatrics in Review, 2013; 34(5):203-215.

[8]. Olokoba AB, Obateru OA, Olokoba LB. Type 2 Diabetes Mellitus: A Review Of Current Trends. Oman Medical Journal, 2012; 27(4):269-273.

[9]. Cleveland Clinic: Diabetes: An Overview

[10].Mahnaz Rezaei-Kelishadi, Mohsen Nuri, Zahra Erfani, Abbasali Palizban, Rahmatollah Parandin. Control, Management and Treatment Of Diabetes Using Modern Drug Delivery Systems And Special Properties Of Nanoparticles. Journal Of Biology And Today's World, 2014;3(9):206-211

[11].Minyahil A. Woldu, Jimma L. Lenjisa. Nanoparticles and The New Era In Diabetes Management. International Journal of Basic \& Clinical Pharmacology, 2014;3(2):277-284.

[12].Gupta Ritika. Diabetes Treatment by Nanotechnology. J Biotechnol Biomater, 2017;7(3):1-2.

[13].Simos Yannis V, Patiala Michaela, et Al. Trends Of Nanotechnology In Type 2 Diabetes Mellitus Treatment. Asian Journal Of Pharmaceutical Sciences, 2021;16(1):62-76. 